\title{
Study on the Mechanism of Atropine in the Treatment of Septic Shock
}

\author{
Xin Yan \\ Shenyang Pharmaceutical University, Benxi 117000, China
}

\begin{abstract}
Atropine (atropinol) is a typical anticholinergic drug that competes for $\mathrm{M}$ receptors. Large doses of atropine can expand blood vessels, relieve small vasospasm, improve microcirculation as well as cardiovascular function, and inhibit thromboxane formation. It is an ideal drug for the treatment of septic shock that are worthy of clinical application. In recent years, studies have found that the main mechanisms of atropine's anti-infective shock include inhibiting calcium ion overload, improving microcirculation disorders, inhibiting thromboxane formation, reducing the release of inflammatory mediators, and inhibiting the release of glutamate. The research progress of the pharmacological effects and mechanisms of atropine in the treatment of septic shock is summarized, and it provides a basis for the in-depth study of atropine.
\end{abstract}

Keywords: Atropine; Septic shock; Improving microcirculation

Publication date: May, 2021; Publication online: 31 May, 2021

*Corresponding author: Xin Yan, 1033826370@qq.com

\section{Introduction}

Atropine exists in natural plants such as hyoscyamine, belladonna and datura. Datura leaves were used to treat asthma in the $17^{\text {th }}$ century. In 1831 , Mein separated and purified atropine crystals. So far, atropine has a history of more than four hundred years. Atropine is a typical anticholinergic drug that competes for $\mathrm{M}$ receptors. Modern medical researches have found that atropine has pharmacological effects such as improving microcirculation, treating septic shock and myopia, regulating gastrointestinal and bronchospasm, inhibiting glandular secretion as well as anti-herpes virus.

Septic shock is a common emergency and severe disease in the emergency department, and its mortality rate is on the rise. How to treat septic shock effectively and safely has always been a hot issue in the field of pharmacy. Septic shock is a systemic inflammation. In the process of septic shock, toxins or pathogenic microorganisms invade the tissue cells of the whole body through the blood, activate the immune system and host cells, thereby forming endogenous inflammatory mediators and causing cytokine infection to persist low blood pressure, thereby causing blood circulation failure. The organs and systems of the body have a serious impact. In recent years, researchers have conducted in-depth studies on the effects of atropine and other $M$ receptor-competing anticholinergic drugs in the treatment of septic shock and the mechanism of action, and found that atropine has the pharmacological effects of improving microcirculation and treating septic shock, and its mechanism of action including inhibition of calcium ion overload, improvement of microcirculation disorders, inhibition of thromboxane formation, reduction of the release of inflammatory mediators, inhibition of the release of glutamate and so on. This article reviews the latest research progress of atropine in the treatment of septic shock and its possible mechanism, and provides a basis for the further research and clinical application of atropine.

\section{Effects of atropine}

\subsection{Inhibiting calcium ion overload}

Calcium ion is an important molecule for cells to maintain normal life activities. It mainly participates in various physiological activities such as the relaxation and contraction activities of cells in the body, the expression of proteins, signal transduction, and the release of neurotransmitters. Myocardial ischemia and reperfusion will lead to an increase in the production of reactive oxygen 
species, which will oxidize the proteins in cells and mitochondria and destroy their functions. It is currently believed that there are many proteins involved in the formation of calcium overload, such as RyR2, $\mathrm{Na}+/ \mathrm{Ca} 2+$ exchanger, and cell membrane Ca2+-ATPase. Mitochondria act as energy-supply organelles to provide energy for ion exchange. The increase of reactive oxygen species damages the membrane structure of mitochondria, which in turn reduces the ability of ion exchange. When the ion exchange capacity decreases, the $\mathrm{Na}+-\mathrm{Ca} 2+$ exchange weakens, and a large amount of $\mathrm{Ca} 2+$ enters the cell, which causes the calcium ion in the cell to be overloaded. Excessive calcium ions activate phospholipase A2, and membrane phospholipids are degraded to produce arachidonic acid. At the same time, inflammatory mediators such as nitric oxide, leukotrienes and prostaglandins produced by the metabolism of arachidonic acid also increase correspondingly, which reduces cell resistance, hypoxia, ischemia, and endotoxin tolerance as well as the phagocytic function of the reticuloendothelial system ${ }^{[1]}$. In addition, the overloaded calcium ions will enter the mitochondria, which will hinder the synthesis of ATP, which will eventually lead to cell death.

Anisodamine can be used as a special calcium channel inhibitor and participates in the pathological process of calcium ion elevation in the early stage of ischemia. Anisodamine reduces myocardial damage and improves heart function by blocking calcium channels during ischemia. Studies have shown that through myocardial ischemia and reperfusion in rats, compared with the control group, the level of ROS in the serum of $I / R$ model rats was significantly increased. Compared with the I/R model group, the $\mathrm{I} / \mathrm{R}$-induced increase in reactive oxygen species was significantly inhibited after anisodamine treatment. Another study showed that after myocardial ischemia and reperfusion in rabbits, the ICa-L current intensity of ventricular myocytes was significantly increased. If anisodamine pretreatment is used, the rising ICa-L current intensity can be reduced $^{[2]}$. Atropine and hyoscyamine are both cholinergic receptor blockers and have similar pharmacological effects, which indirectly proves that atropine can reduce this abnormally elevated brain $\mathrm{Ca} 2+$ content to a control level.

2.2 Expanding microcirculation blood vessels and improve microcirculation disorders

The ultimate goal of septic shock treatment is to improve the microcirculation disorder, restore the function of the microcirculation, so as to restore the oxygen supply to maintain the cell function. In order to achieve significant results in clinical treatment, the pathophysiological mechanism of microcirculation disorders must be considered $^{[3]}$. Studies have shown that changes in microcirculation may include reduced nitric oxide (NO) availability and decreased red blood cell filtration index and blood viscosity.

First of all, nitric oxide is the main regulator for maintaining vascular tone and homeostasis. Nitric oxide is produced by nitric oxide synthase (ENOS) and plays an important role in the process of regulating microcirculation. Studies have shown that the bioavailability of nitric oxide is related to the uncoupling of nitric oxide synthase, either the degree of L-arginine transport decreases or the expression of arginine utilization enzymes (such as arginase) increases, eventually leads to uncoupling of nitric oxide synthase ${ }^{[4]}$.

Secondly, during septic shock, tissue cells are hypoxic-ischemic, the ability of red blood cells to release nitric oxide is severely reduced, red blood cells lose their ability to deform, and blood viscosity increases. Studies have found that high-dose anisodamine (20 $\mathrm{mg} \cdot \mathrm{kg}-1)$ carotid perfusion can effectively inhibit the increase in hippocampal nitric oxide (NO) value of rats with cerebral ischemia-reperfusion injury, and reduce the blood flow caused by it. Disorder effect, and then improve microcirculation disorders ${ }^{[5]}$. Sun Jun et al. ${ }^{[6]}$ proved through experiments that anisodamine has an inhibitory effect on lipopolysaccharide binding to vascular endothelial cells and inducing NO synthesis. After the rats were injected with anisodamine, the red blood cell filtration index and blood viscosity decreased, and the deformability of red blood cells was greatly improved. The pharmacological effects of atropine and anisodamine are similar, indicating that atropine can enhance red blood cell deformability, reduce blood viscosity, increase the ability of red blood cells to pass through the capillary wall, speed up blood flow, and improve microcirculation.

\subsection{Inhibiting the formation of thromboxane}

Thromboxane A2 is a powerful inducer of vasoconstriction, platelet aggregation and bronchoconstriction. Prostaglandin endoperoxide (PGG2/PGH2) is the precursor of prostaglandin and thromboxane $\mathrm{A} 2^{[7]}$. In the presence of thromboxane A2 synthase inhibitors, prostaglandin endoperoxide (PGG2/PGH2) may accumulates in large 
quantities, thus exerting biological activity. The canine coronary artery thrombosis model showed that thromboxane A2 synthase inhibitors interact with prostaglandin endoperoxide receptor antagonists, confirming that prostaglandin endoperoxide has a certain effect on thromboxane A2 synthase in the body. The inhibitory effect of serotonin has certain guiding significance for the clinical treatment of septic shock.

There are many natural antagonists in the human body, which play diametrically opposite effects in the human body, such as insulin and glucagon. Thromboxane A2 and prostaglandins are also natural antagonists. They are important active substances that jointly regulate blood flow, blood flow distribution and vascular wall tension. Prostacyclin is currently considered to be a physiological antagonist of thromboxane A2 (TXA2) ${ }^{[8]}$. It has the effects of promoting aggregation and contraction of blood vessels, relaxing blood vessels, inhibiting platelet aggregation and accelerating blood flow. Under normal circumstances, the two maintain a dynamic balance in the tissue to maintain the stability of the microcirculation. When there is too much thromboxane A2 synthetase and the generated thromboxane A2 far exceeds the content of prostaglandin, the blood vessels in the body are strongly contracted, the peripheral resistance increases, and the blood flow speed slows down, resulting in microcirculation disorders.

Zhou Daixing et al.[9] found that early application of anisodamine treatment to rabbits after cerebral ischemia and reperfusion can significantly reduce the content of thromboxane A2 in brain tissue during reperfusion. This indicates that anisodamine can reduce the synthesis of thromboxane A2 during cerebral ischemia and reperfusion, regulate the dynamic balance of thromboxane $\mathrm{A} 2$ and prostaglandin, and improve cerebral circulation. Therefore, atropine may reduce the synthesis of thromboxane $\mathrm{A} 2$ by inhibiting thromboxane A2 synthase or cyclooxygenase, and expand the microcirculation blood vessels, inhibit the aggregation of platelets, accelerate the blood flow rate, and thereby change the microcirculation disorder.

\subsection{Reducing the release of inflammatory mediators and improve microcirculation}

The inflammatory response mainly involves the participation of inflammatory cells, including macrophages, lymphocytes and neutrophils. In the process of inflammatory response, these inflammatory cells will release special active substances, mainly including pro-inflammatory cytokines, vasoactive peptides and polypeptides. These substances will further cause damage to tissue cells, leading to microcirculation disorders.

Macrophages play an important regulatory role in the process of inflammatory response. When macrophages are stimulated, they immediately produce inflammatory signals that promote the inflammatory response, and then macrophages produce a large number of cytokines, chemokines and growth factors to promote the occurrence of inflammation, such as nitric oxide (NO), interleukin-1 (IL-1), tumor necrosis factor (TNF), etc. At the same time, activated macrophages can produce nitrogen and oxygen intermediates and reactive oxygen species, including superoxide and nitric oxide, which are highly toxic to the body, and can also cause high damage to adjacent tissue cells and cause abnormal inflammation. At the same time, the central nervous system can regulate the production of pro-inflammatory cytokines through the vagus nerve. The neurotransmitter acetylcholine of the vagus nerve inhibits the production of pro-inflammatory cytokines by macrophages stimulated by endotoxin through $\alpha 7 \mathrm{nAChR}$. Studies have shown that anisodamine can block muscarinic receptors and promote the binding of endogenous acetylcholine to $\alpha 7 \mathrm{nAChR}^{[10]}$. When endogenous acetylcholine is combined with $\alpha 7 \mathrm{nAChR}$, it can inhibit the production and release of pro-inflammatory cytokines in the ischemic shock model and control septic shock.

ET-1 is a vasoactive peptide, which can stimulate the release of a variety of inflammatory mediators and produce a strong and long-lasting vasoconstrictor effect. ET-1 has a powerful contraction effect on the cerebral arteries, causing a sharp drop in cerebral blood flow, leading to infarction and impaired nerve function. Tang Yan et al. ${ }^{[11]}$ found that the content of ET-1 in the ischemia group was significantly higher than that in the sham operation group, and the content of ET-1 in the reperfusion control group continued to increase and was significantly higher than that in the sham operation group, indicating that ET-1 was involved in cerebral ischemia reperfusion injury process. This proves that atropine and other $\mathrm{M}$ choline receptor blockers can effectively reduce the secretion and release of ET-1, thereby reducing the secretion and release of inflammatory mediators, increasing the release of $\mathrm{NO}$, inhibiting excessive contraction of blood vessels, increasing blood flow, and releasing Vasospasm.

\subsection{Inhibiting the release of glutamate}


Glutamate is an excitatory amino acid, and its receptor exists on the surface of platelets. When it is over-released, glutamate can induce the mRNAs present in platelets to synthesize thrombi to form peptide plasminogen activator inhibitor-1 (PAI-1) and hypoxia-inducible factor-2 $\alpha$ (HIF-2 $\alpha$ ), while glutamate can increase the transmembrane potential of mitochondria, thereby generating reactive oxygen species and inducing the activation of AMP-activated protein kinase in platelets ${ }^{[12]}$. Hypoxia-inducible factor- $2 \alpha$ (HIF-2 $\alpha$ ) consists of an oxygen-sensitive $\alpha$ subunit and a constitutively expressed $\beta$ subunit, which plays a vital role in oxygen homeostasis. Under hypoxic conditions, proline hydroxylase cannot hydroxylate HIF- $2 \alpha$, making HIF-2 $\alpha$ unable to stabilize, but HIF- $2 \alpha$ can also be stabilized by non-hypoxic stimulation, such as thrombin, which can cause thrombus The formation of microcirculation barriers.

Zhan Chengye et al. ${ }^{[13]}$ conducted experimental studies on rabbits with global cerebral ischemia and reperfusion and found that the content of glutamate decreased significantly during the period of ischemia-reperfusion in rabbits, and the application of anisodamine in the early stage of reperfusion could inhibit glutamate. Excessive release of serotonin reduces the formation of microthrombi, thereby improving microcirculation. In the same way, atropine and anisodamine are both effective $\mathrm{M}$ cholinergic receptor blockers and have the same pharmacological effects to a certain extent, which can indicate that atropine effectively inhibits the release of glutamate during septic shock. , Which reduces the formation of microthrombosis, which plays a vital role in the treatment of septic shock.

\section{Summary and outlook}

Septic shock is a serious hazard to human life and health, and its mortality rate is increasing year by year. Although relevant methods have been used in clinical treatment, the treatment effect is not satisfactory. Therefore, the treatment of septic shock has become a research hotspot at home and abroad. As one of the $\mathrm{M}$ choline receptor blockers, atropine has obvious therapeutic effects on septic shock, and its clinical value needs to be further developed.

In recent years, studies at home and abroad have found that atropine is an ideal drug for the treatment of septic shock. It can block norepinephrine alpha receptors, block the synthesis of adrenaline, inhibit platelet aggregation, and reduce the release of inflammatory mediators. Atropine is an important means to treat septic shock.

\section{References}

[1] Innes JK, Calder PC. Omega-6 fatty acids and inflammation[J]. Prostaglandins Leukot Essent Fatty Acids, 2018,132:41-48.

[2] Ding X, Fu XH, Zhao YY, et al. Effects of anisodamine on L-calcium channel currents in ventricular myocytes after ischemia-reperfusion in rabbits $[\mathrm{J}]$. Lingnan Journal of Cardiovascular Diseases, 2010-08-28.

[3] Slovinski AP, Hajjar LA, Ince C. Microcirculation in Cardiovascular Diseases[J]. Cardiothorac Vasc Anesth, 2019,33(12):3458-3468.

[4] Förstermann U, Sessa WC. Nitric oxide synthases: regulation and function[J]. Eur Heart J,2012,33(7):829-37

[5] Pang T, Xu T. The protective mechanism of $\mathrm{M}$ choline receptor blockers on cerebral ischemia-reperfusion injury[J]. Journal of Xuzhou Medical College, 2006(06):592-595.

[6] Sun J, Yao XJ, Zhao DH. Inhibition of anisodamine on lipopolysaccharide binding to vascular endothelial cells and inducing NO synthesis[J]. Journal of the Fourth Military Medical University, 2001(12): 1082-1084.

[7] Fitzgerald DJ, Fragetta J, FitzGerald GA. Prostaglandin endoperoxides modulate the response to thromboxane synthase inhibition during coronary thrombosis $[\mathrm{J}]$. Clin Invest, 1988,82(5):1708-13

[8] de Leval X, Hanson J, David JL, et al. New developments on thromboxane and prostacyclin modulators part II: prostacyclin modulators $[\mathrm{J}]$. Curr Med Chem, 2004 ,11(10):1243-52.

[9] Zhou DX, Deng PZ. The effect of anisodamine on the metabolism of TXA_2 and PGI_2 in the brain tissue of rabbits with global cerebral ischemia and reperfusion[J] Chinese Emergency Medicine, 2000(12): 9-10.

[10] Sun L, Zhang GF, Zhang X, et al. Combined administration of anisodamine and neostigmine produces anti-shock effects: involvement of $\alpha 7$ nicotinic acetylcholine receptors $[\mathrm{J}]$. Acta Pharmacol Sin,2012,33(6):761- 6.

[11] Tang Y, Yang GT, Jiang CH, Wang PH, Deng PZ. Effects of anisodamine on nitric oxide endothelin-1 and energy metabolism in rats with acute global cerebral ischemia-reperfusion injury[J]. Chinese Emergency Medicine , 2000(04):201-203. 
[12] Gautam D, Tiwari A, Nath Chaurasia R, et al. Glutamate induces synthesis of thrombogenic peptides and extracellular vesicle release from human platelets[J]. Sci Rep,2019,9(1):8346.
[13] Zhan CY, Deng PZ. The effect of anisodamine on the release of excitatory amino acids and survival during acute global cerebral ischemia and reperfusion in rabbits[J]. Chinese Emergency Medicine, 1999(03): 3-5. 